\title{
Improving ability of tele-operators to complete progressively more difficult mobile robot paths using simple expert systems and ultrasonic sensors
}

\author{
David A. Sanders and Fasper Graham-Fones \\ Mechanical and Design Engineering, University of Portsmouth, Portsmouth, UK, and \\ Alexander Gegov \\ School of Computing, University of Portsmouth, Portsmouth, UK
}

\begin{abstract}
Purpose - The purpose of this paper is to describe the use of simple expert systems to improve the performance of tele-operated mobile robots and ultrasonic sensor systems. The expert systems interpret data from the joystick and sensors and identify potentially hazardous situations and then recommend safe courses of action so that tele-operated mobile-robot tasks can be completed more quickly.

Design/methodology/approach - The speed of a tele-operator in completing progressively more complicated driving tasks is investigated while using a simple expert system. Tele-operators were timed completing a series of tasks using a joystick to control a mobile robot through a simple expert system that assisted them with driving the robot while using ultrasonic sensors to avoid obstacles. They either watched the robot while operating it or sat at a computer and viewed scenes remotely on a screen from a camera mounted on the robot. Tele-operators completed tests with the simple expert system and the sensors connected. The system used an umbilical cable to connect to the robot.

Findings - The simple expert systems consistently performed faster than the other systems. Results are compared with the most recently published results and show a significant improvement. In addition, in simple environments, tele-operators performed better without a sensor system to assist them but in more complicated environments than tele-operators performed better with the sensor systems to assist.

Research limitations/implications - Simple expert systems are shown to improve the operation of a tele-operated mobile robot with an obstacle avoidance systems fitted.

Practical implications - Tele-operated systems rely heavily on visual feedback and experienced operators. This paper investigates how to make tasks easier. Simple expert systems are shown to improve the operation of a tele-operated mobile robot. The paper also suggests that the amount of sensor support should be varied depending on circumstances.

Originality/value - The simple expert systems are shown in this paper to improve the operation of a tele-operated mobile robot. Tele-operators completed tests with the simple expert system and the sensors connected. The results are compared with a tele-operator driving a mobile robot without any assistance from the expert systems or sensors and they show a significant improvement.
\end{abstract}

Keywords Sensors, Robotics, Telecontrol, Ultrasonics, Systems and control theory

Paper type Technical paper

\section{Introduction}

This paper presents simple expert systems (Hudson et al., 1997; Sanders et al., 2009b; Sanders, 2009d) that improve the performance of a tele-operated mobile robot and ultrasonic sensor system. The speed of a tele-operator in completing progressively more complicated driving tasks is investigated and the results are compared with the most recently published results

The current issue and full text archive of this journal is available at www.emeraldinsight.com/0143-991X.htm

Industrial Robot: An International Journal

$37 / 5$ (2010) 431-440

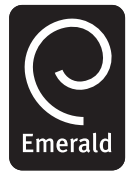

[DOI 10.1108/01439911011063254]
(Sanders, 2009b, 2010a, c). They show a significant improvement.

Insights into trends and new products in the unmanned vehicle industry are described by Bloss $(2008,2009)$ and research was recently presented in this journal concerning the way that a human tele-operator interacts with a mobile robot and ultrasonic sensor system (Sanders, 2010a). The simple expert systems described in this paper improve on that work and results are presented to show that a tele-operator can drive the mobile robot faster using the expert systems. The expert systems identify potentially hazardous situations and recommend safe courses of action so that tele-operated mobile-robot tasks can be completed more quickly.

Tele-operated systems (Stott and Sanders, 2000a, b) tend to rely heavily on visual feedback and experienced human operators and this paper investigates how to make their tasks 
easier by using an expert system to interpret joystick and sensor data. A human tele-operator is still the most accurate source of data about the environment but that source can be impaired by distance, poor vision or restricted view (even with a more local camera). Simple expert systems were therefore created to overcome some of those limitations and to improve the control of the tele-operated mobile robot. The simple expert systems interpret data from a joystick controller (Sanders and Baldwin, 2001) and sensors (Sanders, 2008b) and mix that interpreted data (Stott et al., 1997).

Other work on shared control includes Borenstein and Koren (1990), Conway (1987) and Joh and Yoo (2004) and they are included for completeness.

\section{The sensor systems}

Several sensor systems were considered, including vision (Connolly, 2009; Hopper, 2009; Sanders et al., 1992, 2009a; Sanders, 2010b), infrared (Lee, 2008, 2009), laser (Larsson et al., 2008), GPS (Milanes et al., 2008), force (Sanders, 2007). Ultrasonic ranging was selected to assist because it was simple and robust (Horn and Kreutner, 2009; Rahiman et al., 2009; Sanders and Stott, 1999).

The most recently published ultra-sonic sensor system for a mobile-robot was described by Sanders (2010a, c). This new work uses the same tele-operated mobile-robot base (shown in Figure 1) and the same sensor systems so that results can be directly and easily compared. Two driven wheels were at the front and two trailing casters at the back. A camera could be mounted between the driving wheels and ultrasonic sensor pairs could be mounted over each driving wheel.

The direct link between the mobile robot and joystick was severed and a computer processed control information. Other human-computer interfaces were then considered, including pointers (Sanders et al., 2005; Sanders and Tewkesbury, 2009), computer mice (Bergasa-Suso et al., 2005; Sanders and Rasol, 2001) but a joystick appeared to be the easiest interface to use for this application (Sanders and Stott, 1999).

Figure 1 Bobcat II base unit

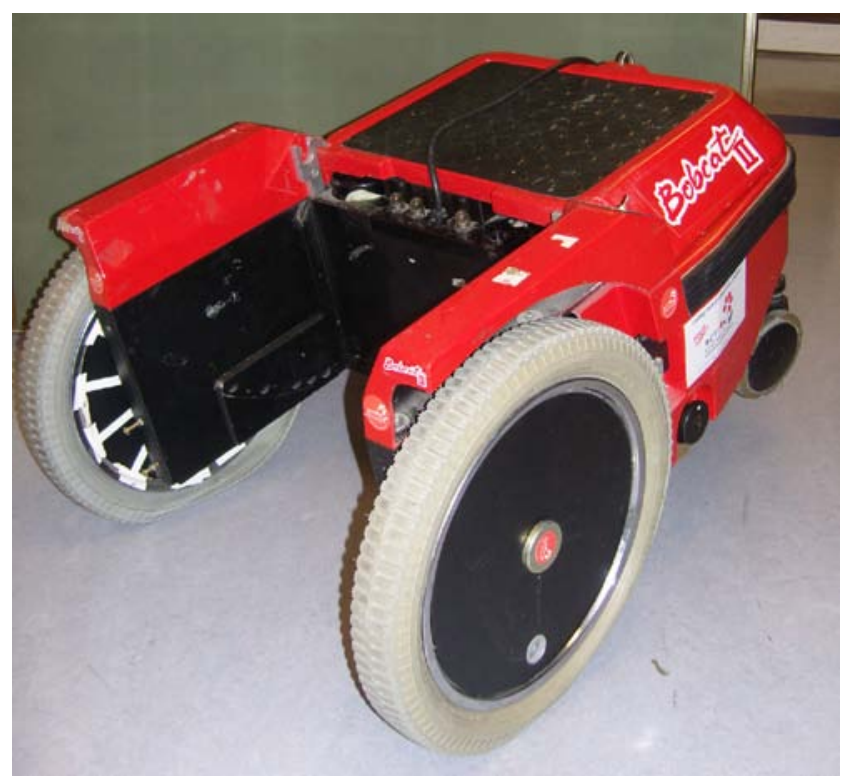

Three modes of operation were possible:

1 Joystick data could be processed and sent to the controller without modification.

2 Sensors were activated and interrogated by the computer and the computer was programmed to modify the mobile-robot path using the most recently published methods.

3 Sensors were activated and interrogated by the computer and the computer was programmed to modify the mobile-robot path using the simple expert systems presented in this paper.

Algorithms applied the following rules:

1 user remained in overall control;

2 systems only modify trajectories when necessary; and

3 movements were smooth and controlled.

An imaginary potential field was generated around objects in response to sensor information to assist users if the mobile robot was approaching an object and could collide.

Ultrasonic sensors tended to be noisy and return misreads. A method for filtering out misreads was selected to improve sensor reliability that was based on histogramic in-motion mapping. Volumes in front of each sensor were divided into a simple grid of three volumes: near, middle and far (as shown in Figure 2).

They were stored as an array in micro-controller memory. When a range was returned, it was classified as near, middle or far. Different numbers of sensors were mounted so that their beams swept the area in front of the mobile robot. The arrangement for two sensors that is described in this paper is shown in Figure 3.

Array elements representing the area in which an object was detected were incremented by a relatively high number (for example 3). Other array elements were decremented by a lower number (for example 1). Arrays typically had a maximum

Figure 2 A simple representation of the envelope of a single ultrasonic sensor

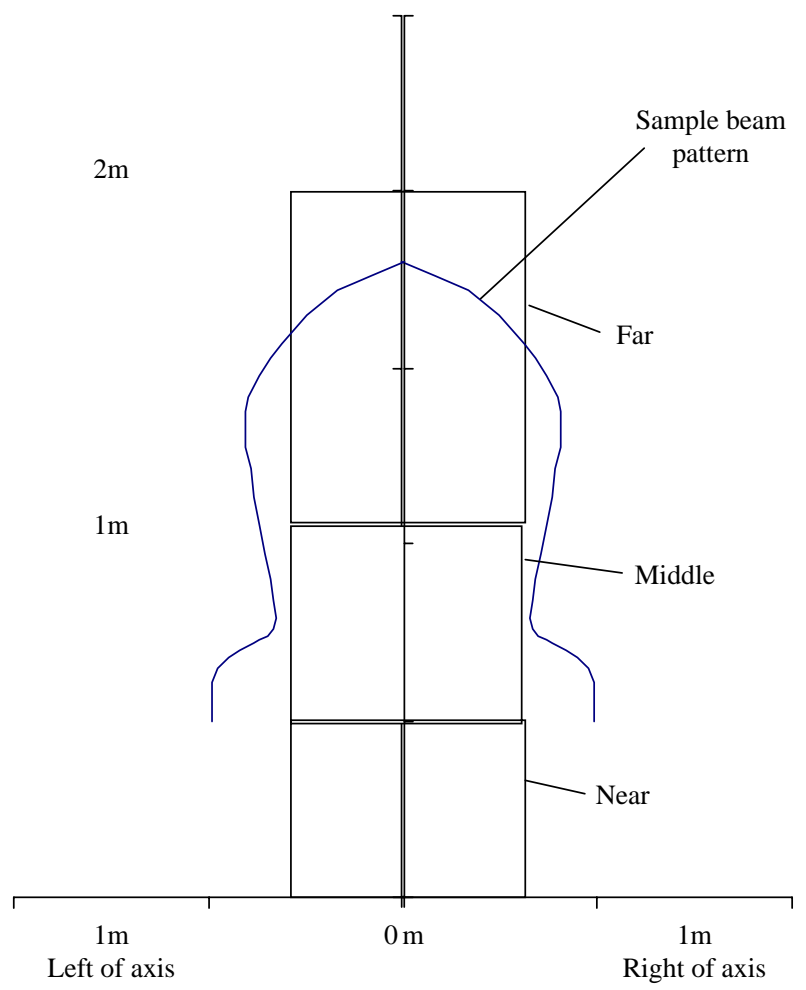


Figure 3 Representation for a two-sensor array

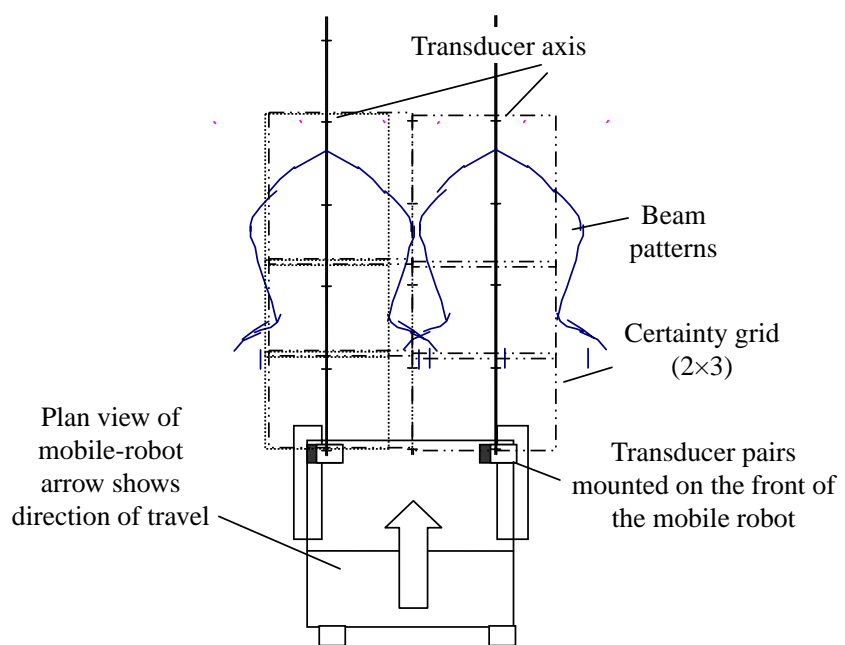

value of 15 and a minimum value of zero. Figure 4 shows an example of the simple three-element histogrammic representation of the environment and the position of an object in the third element causing that element to ramp up. An object occupying a grid element would cause that element to quickly ramp in value to the maximum. Random misreads in the other elements incremented that element temporarily, but the values of the false reads were decremented over time. If the object moved to a different element, the new element quickly ramped up to its maximum value and the old element ramped down.

\section{Algorithms to interpret the joystick}

A standard Penny and Giles Potentiometric joystick was fitted that contained two potentiometers to provide two channels

Figure $4 \mathrm{~A}$ representation of the sensor histograms

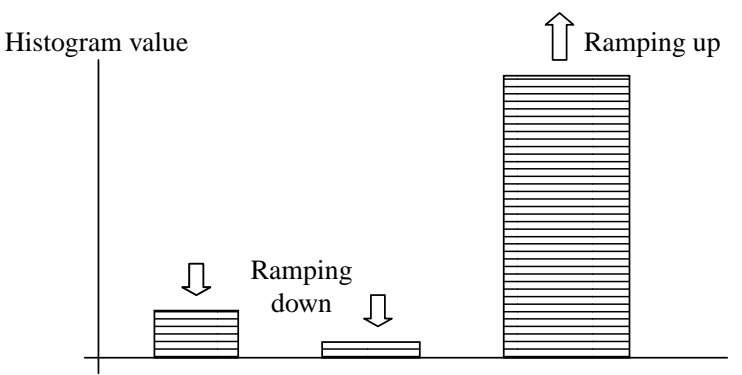

Elements (histograms)

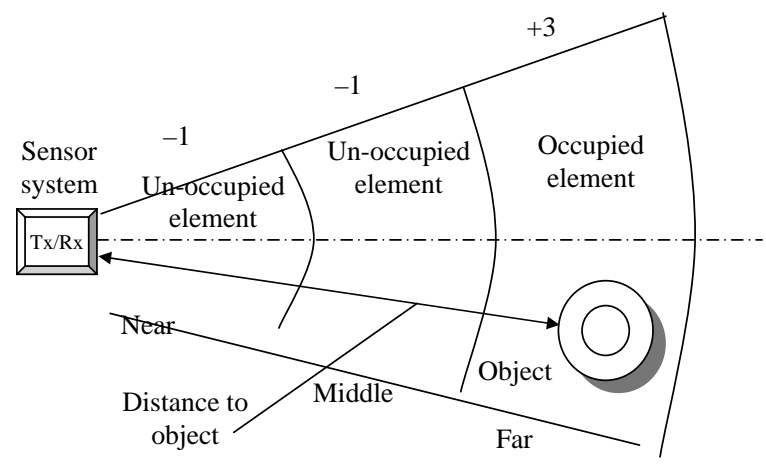

of joystick voltages. Joystick position could be read by an analogto-digital converter as a set of Cartesian co-ordinates. Cartesian co-ordinates however, were not a convenient way to express joystick position. To interpret joystick data in a more convenient manner, Cartesian co-ordinates were converted to polar coordinates using trigonometrical functions and Pythagarus' theorem. Joystick data were now in the form: $|\mathrm{J}| \angle \theta$. Where $|\mathrm{J}|$ was magnitude (or how far the joystick had been pushed) and $\angle \theta$ was the angle of the joystick.

Joystick output was integrated to provide a level of confidence in user intentions. Magnitude could be integrated simply as it was a scalar quantity. The angle of the joystick introduced a directional element and could not be integrated. Joystick angular position was quantified so that intended direction could be estimated. This allowed the algorithms to measure the length of time that a joystick had been held in a consistent direction and helped the new systems to quickly identify the wishes of the tele-operator.

Magnitude and angle were calculated and then used to calculate the sector that the joystick was occupying. The position and confidence of the joystick could be expressed as an array. Each joystick sector contained two array values:

1 "Angle confidence" (0-15) indicated certainty that a joystick was being held in a sector.

2 "Magnitude" indicated joystick position with regard to demanded mobile-robot speed.

A histogrammic representation was then used as a pseudointegrator. If the joystick was held in a position, the array element relating to that position was incremented to raise its overall value. All other array elements could then be decremented to reduce their effect. The array element with the highest value was used as the latest and most confident joystick position. The joystick occupying a joystick array element would cause that element to quickly ramp in value to maximum. Random joystick action in the other elements incremented them temporarily, but values of false reads were decremented each time the system updated. If the joystick moved to a different element, the new element quickly ramped up to maximum and the old element ramped down to the noise level or zero.

Joystick position was represented as a histogram where the highest histogram element represented the most likely direction for the user to be indicating as the desired direction.

A module tested joystick position and angle, and indicated which sector the joystick was occupying. The appropriate element of "angle confidence" was then increased by magnitude 40. All elements were then decreased in magnitude by 30 to decay the un-occupied elements. The occupied element was therefore subject to an increase in magnitude of 10 and all other elements were subject to a decrease in magnitude of 30. This made histogram elements decay rapidly and buildup value more slowly. Ramping and delay weighting factors were determined experimentally by driving the mobile robot with several different weighting factors in operation. In the future, expert systems could automatically set weighting factors after learning about the ability if the user, thus increasing response time for more able users.

\section{The simple expert systems}

The research attempted to introduce some artificial intelligence into the systems (Al-Kasassbeh and Adda, 2009; 
Khan et al., 2009; Sanders et al., 1996; Sanders, 2008c, 2009a, c; Stahl et al., 2009; Urwin-Wright et al., 2002, 2003). The code was written in a mixture of high- and low-level languages and compiled to a single machine level file (Hudson et al., 1996; Sanders, 1993; Sanders et al., 1994, 2001). This file was loaded into non-volatile memory in a micro-controller. The use of an integrated programming environment with access to high-level editing and de-bugging tools assisted in the creation of the prototype systems (Chester, 2006, 2007). A modular structure was adopted to simplify program construction and minimise duplication of code (Hinks et al., 1995, 1996). The structure of the final system was similar to a blackboard-type framework (Sanders et al., 2000; Sanders and Hudson, 2000).

Expert knowledge was acquired from "experts" (Tewkesbury and Sanders, 1994, 1999a, b, 2001); in this case, a human remote fork-lift truck driver (Sanders, 2008a) and an engineer (Harrison and Sanders, 1992). Rules were intended as generative rules of behaviour; given some set of inputs then rules determined what the output should be. A little time was usually needed for a new driver to familiarise themselves with the response of the system to the joystick (Sanders, 1999).

It was important that the system operated in real time in order to assist a tele-operator. There were two real-time inputs to the system: the input device (joystick) and sensors (Goodwin et al., 1997). A user indicated a speed and direction and the sensor system gathered information about the environment. At the highest level, a module called Sensor Expert analysed sensor information and made a recommendation for a path to prevent collisions. Joystick Monitor was responsible for interpreting the wishes of the user. Variables such as joystick position and consistency were examined by Joystick Monitor to assess the desired mobile-robot trajectory. Joystick Monitor sometimes conflicted with Sensor Expert if an obstacle was present. Another expert called Fuzzy Mixer considered both inputs and was responsible for final motor controller outputs. The top-level expert systems consisted of: Fuzzy Mixer, Joystick Monitor, Sensor Expert and Doorway. Doorway was an object avoidance program (Chang and Yamamoto, 2008; Fahimi et al., 2009; Sanders, 1995a, b) that avoided objects through a "distance function" algorithm. Doorway was allowed or over-ridden to affect the trajectory generated by Fuzzy Mixer. Distance to an object measured by the sensors and the current and historical input from the joystick determined how the mobile robot should react. Doorway was effective at turning the mobile robot away from the nearest object, slowing the mobile robot down smoothly as it became closer to objects and centralising the mobile robot between two objects (such as door frames).

Fuzzy Mixer controlled the relationship between the joystick and sensor system and apportioned control to the joystick or sensor system depending on environmental conditions or the wishes of the tele-operator. Instantaneous relationships could be:

- all joystick, no sensors;

- all sensors, no joystick; and

- or somewhere in between.

Algorithms used distance functions to create target values for left and right controller voltages.

To recognise the position of the joystick in order to make an assessment of the wishes of the user, a joystick map was divided into sectors: forward, turn right, turn left, spin right, spin left, stop and back:

1 Factors to increase joystick confidence were:

- joystick agrees with sensor system;

- joystick held in a steady position (consistent); and

- joystick position increased against sensor action.

2 Factors to decrease joystick confidence were:

- joystick - sensor conflict; and

- joystick not held steady.

Sensor Expert applied a set of algorithms to information generated from sensors. There were seven possible actions to decide whether to: do nothing, stop, slow, turn left, spin left, turn right or spin right.

The expert systems were downloaded to the hardware mounted on the mobile robot. Systems were tested by driving the mobile robot in an unstructured but uncluttered environment.

A simplified blackboard framework was used for the overall program structure because the program was easier to control in this structure as all of the main modules communicated with a common blackboard and passed important data to and from the blackboard.

In the case where the joystick and the sensor expert were both indicating "forward", the system set the trajectory as straight-ahead. The sensor system was still interrogated to determine the distance that the mobile robot was from the nearest object. The speed of the mobile robot was reduced as the mobile robot became close to an object.

The algorithm used the SpinLeft or SpinRight command to turn. Although the controller voltage settings were set high (to spin values), the system tended to apply the spin settings for the minimum amount of time required to turn the mobile robot. The mobile robot rarely appeared to spin as the system settings quickly returned to a forward mode. The application of a spin manoeuvre for a limited time simulated a user moving the joystick completely to one side to execute a turn. Observing expert drivers using their joysticks, it became apparent that they often moved the joystick in exaggerated movements (even to perform gentle manoeuvres).

\section{Testing}

Systems were initially tested by driving the mobile robot in an unstructured but uncluttered environment. The response of the mobile-robot system was shown to be safe and fast enough for the mobile robot to navigate itself along a corridor and align itself with a doorway with the joystick held in the forward position. The path that the mobile robot took indicated that Sensor Expert was recommending suitable trajectory changes to the mobile-robot controller.

Mobile-robot systems were then tested in a laboratory and later in a variety of environments. The longest test runs were limited to $30 \mathrm{~m}$ by the lengths of the umbilical cables used. The cables were up to $15 \mathrm{~m}$ long and that allowed a distance of $15 \mathrm{~m}$ out and back. Tele-operated users quickly learnt how the mobile robot responded and learned to apply control signals earlier and to estimate stopping distance.

A set of tests were conducted to compare the speed of human tele-operation with computer-assisted operation in a series of standard environments. Tests were to observe the operation of the system under joint computer and human control and to measure the time taken by: 
- human tele-operators by themselves;

- and then again with the assistance of the most recently published systems; and

- and then again with the assistance of the new systems described in this paper.

For each course, up to six tests took place (two sets of three tests). For each mobile robot, two sets of tests took place without the sensor system or any automatic assistance. Then the tests were repeated with the original sensor system engaged and assistance provided by the computer system and finally the tests were repeated a third time with the new expert systems described in this paper. The two sets of tests were:

1 Tele-operator watching the mobile robot and just using the joystick.

2 Tele-operator remotely watching the space ahead of the mobile robot using a camera mounted on the mobile robot (initially with the view obscured by a mobile screen).

For each test, an obstacle course was set-up in an environment. They were:

- Laboratory. Just two obstacles and a constant open floor space with vertical walls.

- Empty corridors. Flat surfaces and sloping surfaces. Corridor restricted with vertical walls and doorways. Three obstacles offset in a staggered formation.

- Complicated corridors. Flat surfaces and sloping surfaces. Corridor restricted with vertical walls and doorways but with items on the walls (for example radiators and door surrounds). Doorways to pass through. Several obstacles offset in a staggered formation.

- Environments outside. Complex environment with different flat and sloping surfaces. Bounded by different vertical and sloping edges. People walking through and around the environment. Objects in the environment as well as extra obstacles placed in the environment.

Tele-operators were human beings and as such they were variable in their performance and so experiments were repeated several times. That allowed people to learn the systems and to perform at their best in the time available. Testing was regarded as fun by participants and was popular. Competition was encouraged and people tried to beat their best in each test and tried to beat others at the same tests. If a fastest time was achieved by any participant in one set of the tests then they made at least one attempt again at the other test to check that the result was not just due to learning the operation of that particular system. If they managed a fastest time at the other test then they made at least one attempt at the original test. Tests began at a pre-determined and constant start-position (and from a standing start) and timings across the finish lines were measured with both a stopwatch and laboratory clock (an average was taken between the two if there was any discrepancy). Only successful attempts were recorded. That is, any attempt that resulted in a collision was discarded. Figure 5 shows a tele-operator navigating through one of the complicated corridors (with some obstacles) and using the ultrasonic sensor system to assist in steering.

Figure 6 shows the scene from a camera mounted on the front of the robot as it moves though a complicated corridor. The mobile robot is being controlled via the umbilical cable. A tele-operator in a laboratory is guiding the mobile robot assisted by the sensor system on the mobile robot. A researcher with a laboratory digital clock can be seen at the end of the
Figure 5 Tele-operator navigating through one of the complicated corridors using the ultrasonic sensor system to assist in steering

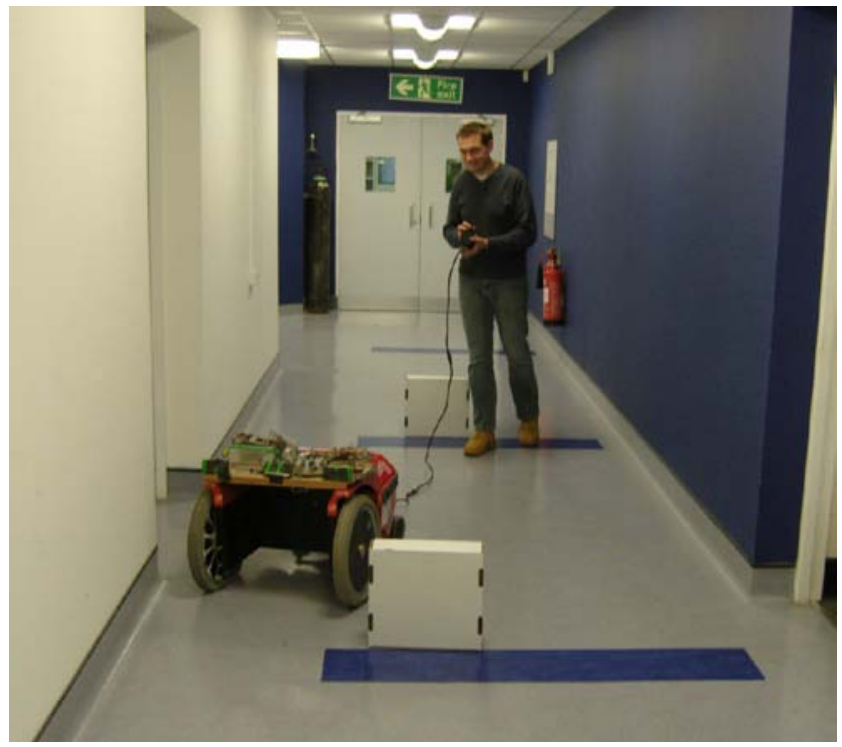

Figure 6 View from a camera mounted on the robot connected via an umbilical cable and moving though a complicated corridor while being assisted by the sensor system

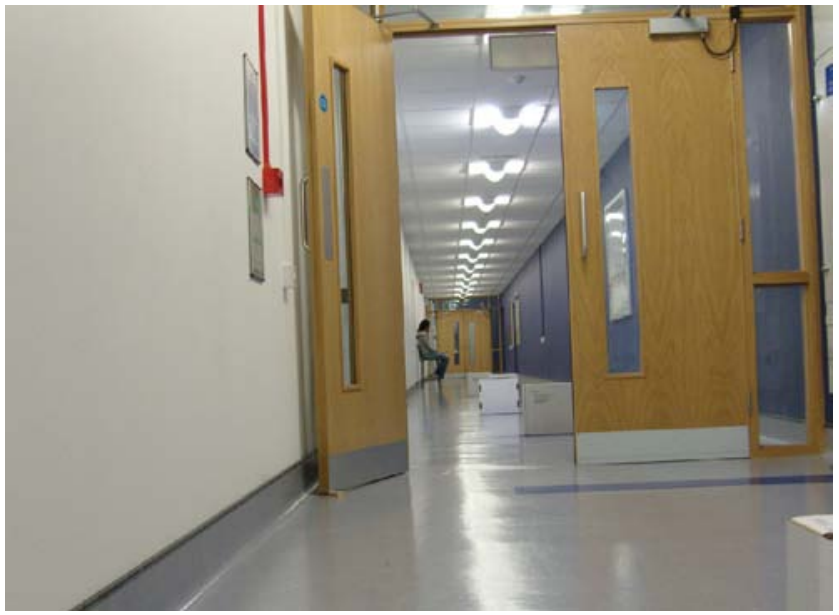

course and another researcher was following the mobile robot with a stop watch.

\section{Results}

The mobile robot successfully negotiated obstacles in various set courses during testing. Assistive computer systems allowed automatic recovery from collision courses. Some chaotic factors existed, for example, trailing casters could throw the mobilerobot off-line and variation in floor surface, slope or wheel position could affect results. Delays between sensor systems providing feedback information and controllers passing results of that feedback information to mobile-robot motors could also cause variations.

The results in Figures 7 and 8 show that the new expert systems consistently performed faster than the most recently 
David A. Sanders, Fasper Graham-Fones and Alexander Gegov

Figure 7 Results from tests when the tele-operator was watching the mobile robot

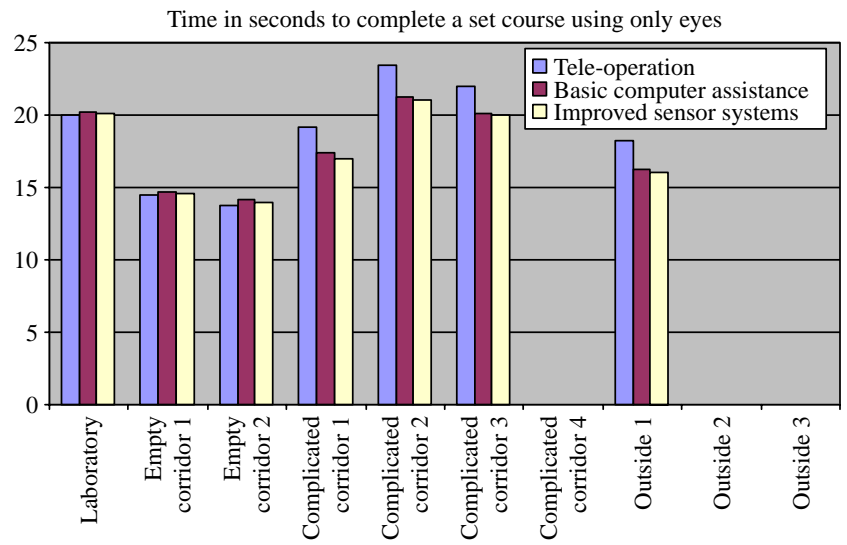

Note: The groups of bars represent the different set courses and the $y$-axis is time in seconds

Figure 8 Results from tests when the camera was mounted on the mobile robot

Time to complete a course using a robot mounted camera

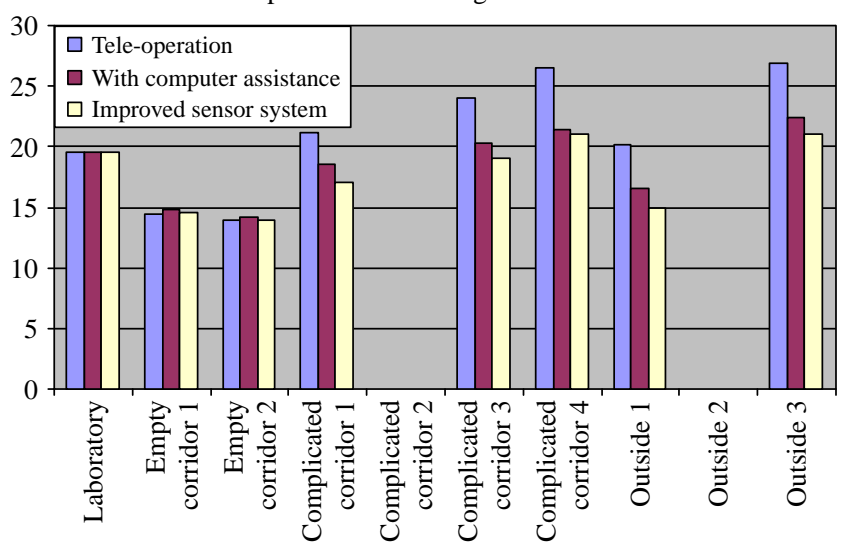

Note: The groups of bars represent the different set courses and the $y$-axis is time in seconds

published system or human tele-operators by themselves. An exception appears to be that in simple environments with few obstacles and wide gaps between them, simple flat and sloping surfaces and straight vertical walls, then human tele-operators consistently performed tasks more quickly without any assistance from the computer systems or sensors. This can be seen in Figures 7 and 8 as the bar charts on the left (simple environments) are the opposite shape to those on the right (more complicated environments). Figure 7 shows the tests completed when the tele-operator was watching the mobile robot (as shown in Figure 5).

That form of results was repeated whether using a camera mounted onto the mobile robot to view the scene ahead (Figure 8) or whether tele-operators watched the mobile robot as they drove it (Figure 7). Human tele-operators were able to drive the robot quickly through wide gaps and observe the situation and make adjustments in plenty of time, without reducing the speed of the mobile robot (and without the need for any sensor or intelligent systems to assist them).
As the environments became more complicated (or the gaps were made smaller) then the human operators found it more difficult to judge the width of the gaps or the successful trajectory of the mobile robot to pass through those gaps. The human tele-operators often had to slow the robot or stop the mobile robot and reverse it to avoid collision. When environments became more complicated, the human teleoperators consistently performed better with assistance from sensors and computer systems (and the new sensor-assisted systems with the expert systems consistently performed faster than the previously published systems). Different surfaces, slopes and boundaries tended to turn robots, thus sensors became most useful in steering through gaps in those cases.

Results became more pronounced as human operators were removed from immersion within the situation and environment. Human operators tended to perform best when they could see the mobile robot and could move around the environment or move with the robot (as shown in Figure 7). When human tele-operators were restricted to using a camera mounted on the mobile robot and observing via a computer screen then the results tended to be slower without the assistance of sensor systems (as shown in Figure 8). With assistance then results were more similar (although still worse as human tele-operators tended to be more cautious with the joystick).

Figures 9-17 show the results from tele-operators watching the mobile robot to tele-operators viewing the area ahead of the mobile robot remotely on a computer screen. Figure 9 shows an interesting result in the simplest and safest environment. The laboratory test only involved driving from one end of the laboratory to the other. In that case, the tele-operators using a remote camera mounted on the mobile robot performed the task more quickly than those watching the robot.

Figure 10 shows the results form an empty corridor (the second most simple course). In this case, the tele-operators completed the course more quickly without any sensor systems to assist them however when the automated systems were used, the new expert system (on the right of the three bars) performed better than the previously published system (in the middle of the three bars). The form of those results are repeated in Figure 11 (a different empty corridor).

The results in Figure 12 are from a more complicated environment. The results have changed a little. Now, the

Figure 9 Results from testing in the laboratory

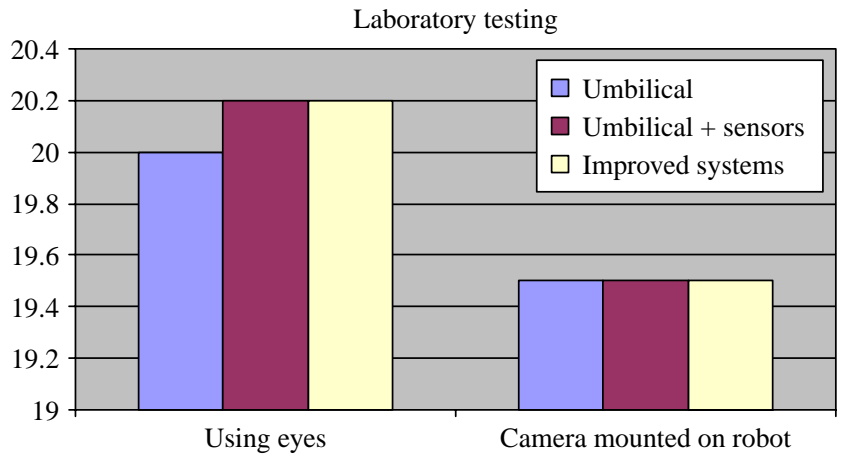

Notes: The group of three bars on the left represent results obtained when directly observing the robot and the group of three bars on the right represent results obtained when watching the view ahead of the robot on a computer screen; the $y$-axis is time in seconds 
Figure 10 Results from testing in empty corridor 1

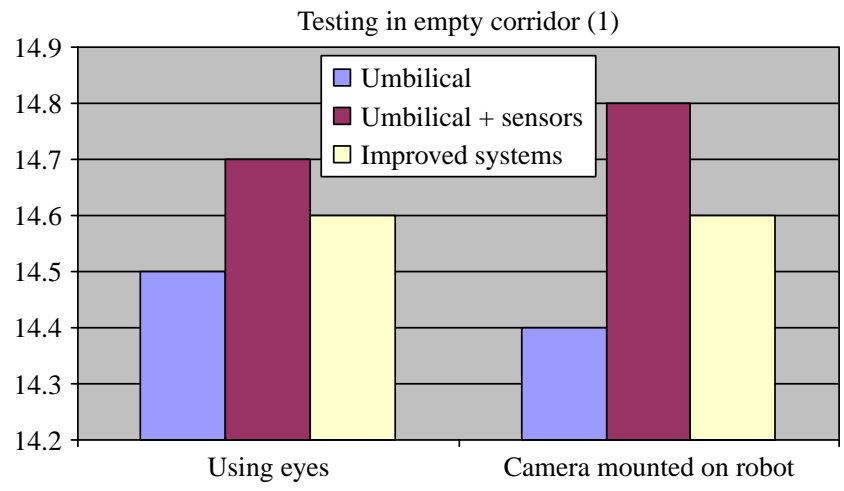

Notes: The group of three bars on the left represent results obtained when directly observing the robot and the group of three bars on the right represent results obtained when watching the view ahead of the robot on a computer screen; the $y$-axis is time in seconds

Figure 11 Results from testing in empty corridor 2

Testing in an empty corridor (2)

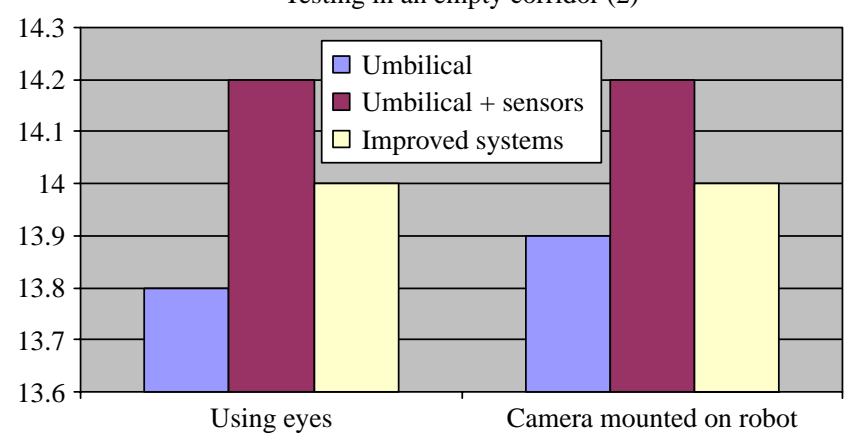

Notes: The group of three bars on the left represent results obtained when directly observing the robot and the group of three bars on the right represent results obtained when watching the view ahead of the robot on a computer screen; the $y$-axis is time in seconds

Figure 12 Results from testing in complicated corridor 1

Testing in a complicated corridor (1)

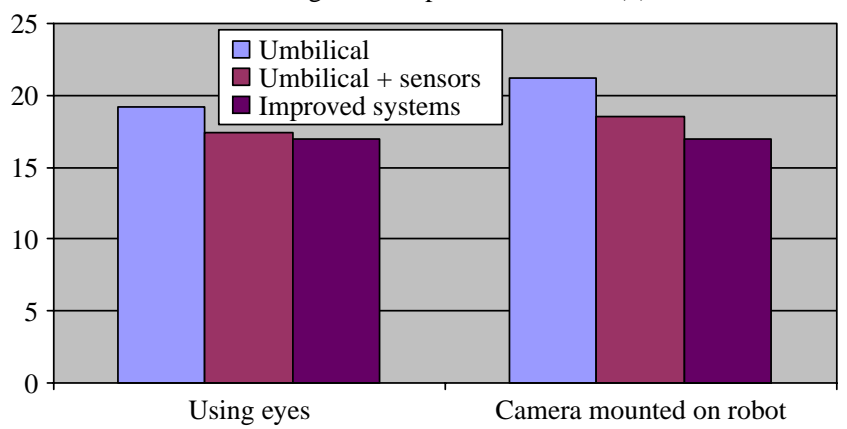

Notes: The group of three bars on the left represent results obtained when directly observing the robot and the group of three bars on the right represent results obtained when watching the view ahead of the robot on a computer screen; the $y$-axis is time in seconds
Volume $37 \cdot$ Number $5 \cdot 2010 \cdot 431-440$

Figure 13 Results from testing in complicated corridor 2

Testing in a complicated corridor (2)

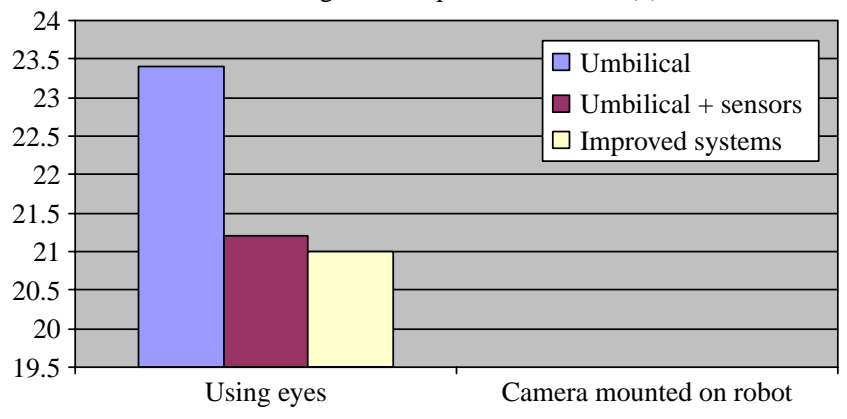

Notes: The group of three bars on the left represent results obtained when directly observing the robot and the group of three bars on the right represent results obtained when watching the view ahead of the robot on a computer screen; the $y$-axis is time in seconds

Figure 14 Results from testing in complicated corridor 3

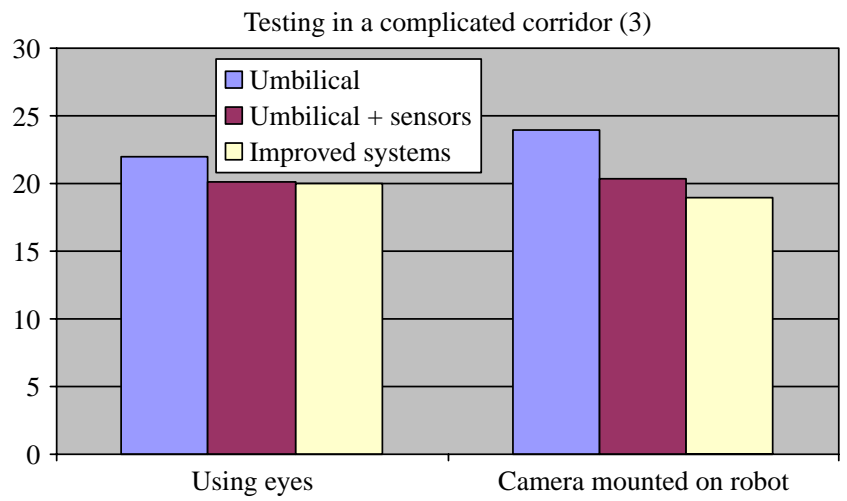

Notes: The group of three bars on the left represent results obtained when directly observing the robot and the group of three bars on the right represent results obtained when watching the view ahead of the robot on a computer screen; the $y$-axis is time in seconds

Figure 15 Results from testing in complicated corridor 4

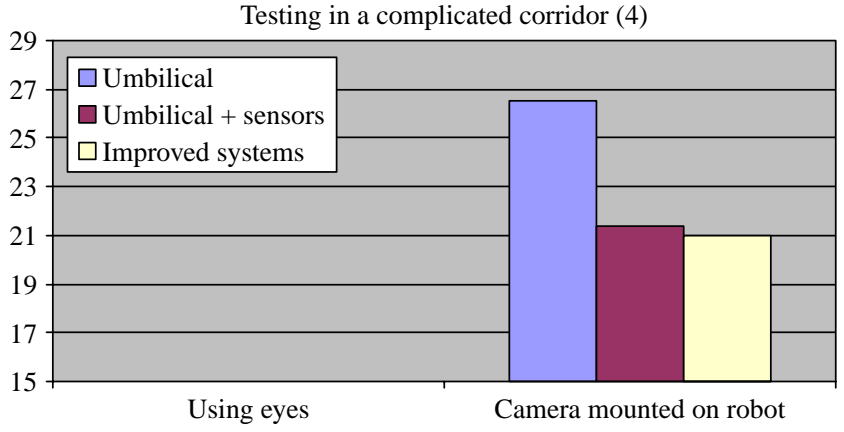

Notes: The group of three bars on the left represent results obtained when directly observing the robot and the group of three bars on the right represent results obtained when watching the view ahead of the robot on a computer screen; the $y$-axis is time in seconds 
Figure 16 Results from testing on outside course 1

Testing outside (1)

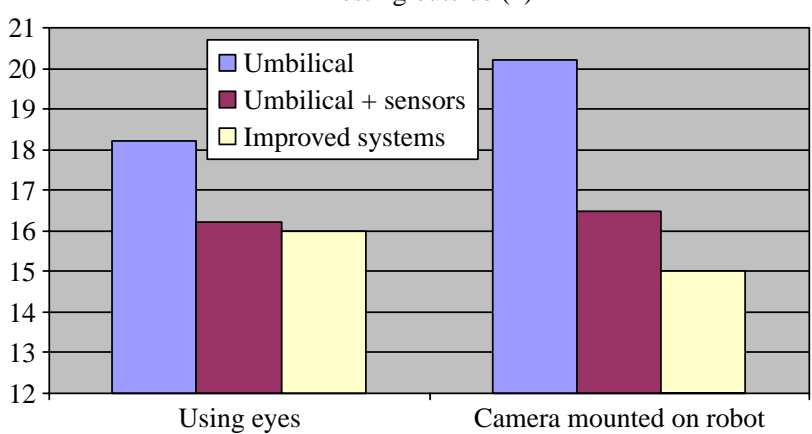

Notes: The group of three bars on the left represent results obtained when directly observing the robot and the group of three bars on the right represent results obtained when watching the view ahead of the robot on a computer screen; the $y$-axis is time in seconds

Figure 17 Results from testing on outside course 3

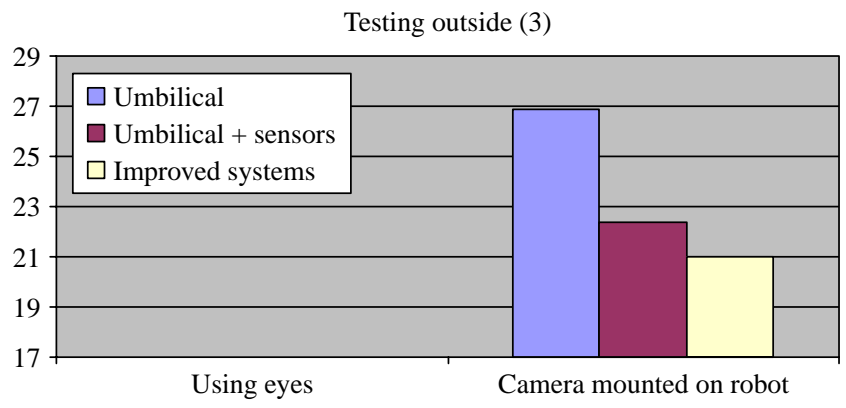

Notes: The group of three bars on the left represent results obtained when directly observing the robot and the group of three bars on the right represent results obtained when watching the view ahead of the robot on a computer screen; the $y$-axis is time in seconds

automated systems consistently perform faster than a human tele-operator alone. Additionally, it can be seen that the new systems described in this paper perform consistently (and significantly) faster than the most recently published system.

That result is repeated in Figure 13, which only shows results when tele-operators were looking at the mobile robot (as in Figure 5) as not enough results were available for statistical comparison when the camera was mounted on the robot. Figure 13 shows the results from a more complicated environment.

The form of the results is repeated in the other more complicated environments shown in Figures 14 and 15. They become more pronounced as the environments become more complicated.

The form of the results is repeated again in the other complicated outdoor environments shown in Figures 16 and 17 .

\section{Discussion and conclusions}

The $t$-test was used to compare means of samples. From each sample, the average (mean) $\chi$ was calculated with a measure of dispersion (range of variation) of data around the sample mean (variance $S^{2}$ ) and thence the standard deviation (S). Having obtained those values, they were then used to estimate population mean $\mu$ and variance $\sigma^{2}$. Each individual set of tests were not statistically significant so that caution was required before generalising the results.

Results were arranged into two sets of replicate data; pairs of results with and without sensor assistance. The paired samples test was used because people (tele-operators) were inherently variable. Pairing removed much of that random variability. When results were analysed using a paired-samples statistical test then results were revealed as statistically significant. The paired-samples statistical test shows the use without a sensor system and with a sensor system to be significantly different at $p<0.05$ (95 per cent probability that the resultant tie differences would not occur by chance alone).

The new simple expert systems performed faster than the previously published methods and systems. In simple environments, tele-operators performed faster without a sensor system to assist them but in more complicated environments than tele-operators performed faster with a sensor system to assist them. As environments became more complicated, human operators could not judge gap widths or the successful trajectory of the mobile-robot to pass through those gaps. Tele-operators often had to slow the robot or stop the mobile-robot and reverse it to avoid collision. When the environment became more complicated, tele-operators consistently performed better with the assistance of the sensors and computer systems. There tended to be some narrower gaps in more complicated routes and as the gaps reduced in width, using assistive systems consistently performed faster than human tele-operators alone. The narrower gaps accounted for some of the improvement when the tele-operators were assisted by a sensor system.

Results became more pronounced in the more complicated environments as human operators were removed from immersion within the situation. In addition, when people were walking near to mobile robots and sensor systems were not engaged, human tele-operators tended to stop mobile robots (and sometimes to abort missions if they felt they had lost their chance to beat their best time). When they were assisted by sensors, they tended to drive on because they knew the mobile robot could avoid people most of the time.

\section{References}

Al-Kasassbeh, M. and Adda, M. (2009), "Network fault detection with Wiener filter-based agent", fournal of Network and Computer Applications, Vol. 32 No. 4, pp. 824-33.

Bergasa-Suso, J., Sanders, D.A. and Tewkesbury, G.E. (2005), "Intelligent browser-based systems to assist internet users", IEEE Transactions (E), Vol. 48 No. 4, pp. 580-5.

Bloss, R. (2008), "Live demonstrations highlight unmanned vehicle show", Industrial Robot, Vol. 35 No. 2, pp. 103-7.

Bloss, R. (2009), "Latest unmanned vehicle show features both innovative new vehicles and miniaturization", Industrial Robot: An International fournal, Vol. 36 No. 1, pp. 13-18.

Borenstein, J. and Koren, Y. (1990), "Tele-autonomous guidance for mobile robots", IEEE Transactions on Systems, Man and Cybernetics, Vol. 20 No. 6, pp. 1437-56. 
Chang, Y.C. and Yamamoto, Y. (2008), "On-line path planning strategy integrated with collision and dead-lock avoidance schemes for wheeled mobile robot in indoor environments", Industrial Robot, Vol. 35 No. 5, pp. 421-34.

Chester, S., Tewkesbury, G., Sanders, D. and Salter, P. (2006), "New electronic multi-media assessment system", WEBIST 2006: Proceedings of the Second International Conference on Web Information Systems and Technologies, Setúbal, pp. 320-4.

Chester, S., Tewkesbury, G., Sanders, D. and Salter, P. (2007), "New electronic multi-media assessment system", Web Information Systems and Technologies, Vol. 1, pp. 414-20.

Connolly, C. (2009), "Machine vision advances \& applications", Assembly Automation, Vol. 29 No. 2, pp. 106-11.

Conway, L. (1987), “Tele-autonomous systems: methods and architectures for intermingling autonomous and tele-robotic technology", Technical Report, Electrical Engineering and Computer Science, Department of EECS, College of Engineering, University of Michigan, Ann Arbor, MI.

Fahimi, F., Nataraj, C. and Ashrafiuon, H. (2009), "Real-time obstacle avoidance for multiple mobile robots", Robotica, Vol. 27, pp. 189-98.

Goodwin, M.J., Sanders, D.A. and Stott, I.J. (1997), "Navigational assistance for disabled wheelchair-users", Fournal of Systems Architecture, Vol. 43 Nos 1-5, pp. 73-9.

Harrison, D. and Sanders, D.A. (1992), "Imagineering promoting creativity in engineering education", Engineering Education, Vol. 2, pp. 45-9.

Hinks, J.W., Cawte, H., Sanders, D.A., Hudson, A. and Dockree, C. (1995), "Model for the prediction of liquid volumetric flow rates in large scale airlift reactors", 3rd International Conference on Water and Waste Water Treatment, BHR Group Conference Series, No. 17, BHR Group, Cranefield, pp. 125-33.

Hinks, J.W., Cawte, H., Sanders, D.A., Hudson, A. and Dockree, C. (1996), "Prediction of flow rates and stability in large scale airlift reactors", Water Science and Technology, Vol. 34 Nos 5/6, pp. 51-7.

Hopper, D. (2009), "The long perspective for robotic vision", Assembly Automation, Vol. 29 No. 2, pp. 122-6.

Horn, O. and Kreutner, M. (2009), "Smart wheelchair perception using odometry, ultrasound sensors, and camera", Robotica, Vol. 27, pp. 303-10.

Hudson, A.D., Sanders, D.A. and Tewkesbury, G.E. (1996), "Simulation of a high recirculation airlift reactor for steady-state operation", Water Science and Technology, Vol. 34 Nos 5/6, pp. 59-66.

Hudson, A.D., Sanders, D.A., Golding, H., Tewkesbury, G.E. and Cawte, H. (1997), "Aspects of an expert design system for the wastewater treatment industry", fournal of Systems Architecture, Vol. 43 Nos 1-5, pp. 59-65.

Joh, J. and Yoo, B.S. (2004), "Tele-operation of a low-cost un-autonomous mobile robot using new fuzzy command smoothing concept", Proceedings of the SICE 2004 Annual Conference, Sapporo, Vol. 1, pp. 45-50.

Khan, A.A., Adda, M. and Adams, C. (2009), "Convergence of terrestrial and satellite mobile communication systems: an operator's perspective", International fournal of Mobile Communications, Vol. 7 No. 3, pp. 308-29.

Larsson, J., Broxvall, M. and Saffiotti, A. (2008), "Laserbased corridor detection for reactive navigation", Industrial Robot: An International fournal, Vol. 35 No. 1, pp. 69-79.
Lee, S. (2008), "Use of infrared landmark zones for mobile robot localization", Industrial Robot, Vol. 35 No. 2, pp. 153-9.

Lee, S. (2009), "Use of infrared light reflecting landmarks for localization”, Industrial Robot, Vol. 36 No. 2, pp. 138-45.

Milanes, V., Naranjo, J.E., Gonzalez, C., Alonso, J. and de Pedro, J. (2008), "Autonomous vehicle based in cooperative GPS and inertial systems", Robotica, Vol. 26, pp. 627-33.

Rahiman, M.H.F., Zakaria, Z., Rahim, R.A. and Ng, W.N. (2009), "Ultrasonic tomography imaging simulation of twophase homogeneous flow", Sensor Review, Vol. 29 No. 3, pp. 266-76.

Sanders, D.A. (1993), "System specifiction 2", Microprocessing and Microprogramming, Vol. 38 Nos 1-5, p. 833.

Sanders, D.A. (1995a), "Real-time geometric modelling using models in an actuator space and Cartesian space", fournal of Robotic Systems, Vol. 12 No. 1, pp. 19-28.

Sanders, D.A. (1995b), "The modification of pre-planned manipulator paths to improve the gross motions associated with the pick and place task", Robotica, Vol. 13, pp. 77-85.

Sanders, D.A. (1999), "Perception in robotics", Industrial Robot, Vol. 26 No. 2, pp. 90-2.

Sanders, D.A. (2007), "Force sensing", Industrial Robot, Vol. 34 No. 4, p. 268.

Sanders, D.A. (2008a), "Controlling the direction of 'walkie' type forklifts and pallet jacks on sloping ground", Assembly Automation, Vol. 28 No. 4, pp. 317-24.

Sanders, D.A. (2008b), "Environmental sensors and networks of sensors", Sensor Review, Vol. 28 No. 4, pp. 273-4.

Sanders, D.A. (2008c), "Progress in machine intelligence", Industrial Robot, Vol. 35 No. 6, pp. 485-7.

Sanders, D.A. (2009a), "Ambient-intelligence, rapidprototyping and where real people might fit into factories of the future", Assembly Automation, Vol. 29 No. 3, pp. 205-8.

Sanders, D.A. (2009b), "Comparing speed to complete progressively more difficult mobile robot paths between human tele-operators and humans with sensor-systems to assist", Assembly Automation, Vol. 2 No. 3, pp. 230-48.

Sanders, D.A. (2009c), "Introducing AI into MEMS can lead us to brain-computer interfaces and super-human intelligence", Assembly Automation, Vol. 29 No. 4, pp. 309-12.

Sanders, D.A. (2009d), "Recognizing shipbuilding parts using artificial neural networks and Fourier descriptors", Proceedings of Institution of Mechanical Engineers Part B fournal of Engineering Manufacture, Vol. 223 No. 3, pp. 337-42.

Sanders, D.A. (2010a), "Analysis of the effects of time delay on the tele-operation of a mobile robot in various modes of operation", Industrial Robot, Vol. 36 No. 6, pp. 570-84.

Sanders, D.A. (2010b), "A robotic welding system using image processing techniques and a CAD model to provide information to a multi-intelligent decision module", Assembly Automation, Vol. 30 No. 4.

Sanders, D.A. (2010c), "Comparing ability to complete simple tele-operated rescue or maintenance mobile robot tasks with and without a sensor system", Sensor Review, Vol. 30 No. 1, pp. 40-50.

Sanders, D.A. and Baldwin, A. (2001), "X-by-wire technology", Total Vehicle Technology: Challenging Current 
Thinking, Department of Mechanical and Manufacturing Engineering, University of Portsmouth, Portsmouth, pp. 3-12.

Sanders, D.A. and Hudson, A.D. (2000), "A specific blackboard expert system to simulate and automate the design of high recirculation airlift reactors", Mathematics $\mathcal{E}$ Computers in Simulation, Vol. 53 Nos 1-2, pp. 41-65.

Sanders, D.A. and Rasol, Z. (2001), "An automatic system for simple spot welding tasks", Total Vehicle Technology: Challenging Current Thinking, Department of Mechanical and Manufacturing Engineering, University of Portsmouth, Portsmouth, pp. 263-72.

Sanders, D.A. and Stott, I.J. (1999), "A new prototype intelligent mobility system to assist powered wheelchair users", Industrial Robot, Vol. 26 No. 6, pp. 466-75.

Sanders, D.A. and Tewkesbury, G.E. (2009), "A pointer device for TFT display screens that determines position by detecting colours on the display using a colour sensor and an artificial neural network", Displays, Vol. 30 No. 2, pp. 84-96.

Sanders, D.A., Cawte, H. and Hudson, A.D. (2001), "Modelling of the fluid dynamic processes in a highrecirculation airlift reactor", International fournal of Energy Research, Vol. 25 No. 6, pp. 487-500.

Sanders, D.A., Harris, P. and Mazharsolook, E. (1992), "Image modelling in real-time using spheres and simple polyhedra", Proceedings of the 4th International Conference on Image Processing E Its Applications, Vol. 354, pp. 433-6.

Sanders, D.A., Lambert, G. and Pevy, L. (2009a), "Pre-locating corners in images in order to improve the extraction of Fourier descriptors and subsequent recognition of shipbuilding parts", Proceedings of Institution of Mechanical Engineers Part B - Fournal of Engineering Manufacture, Vol. 223 No. 9, pp. 1217-23.

Sanders, D.A., Haynes, B., Tewkesbury, G.E. and Stott, I.J. (1996), "The addition of neural networks to the inner feedback path in order to improve on the use of pre-trained feed forward estimators", 2nd European Robotics and Intelligent Systems Conference (EURISCON 94), Malaga, Vol. 41, pp. 461-72.

Sanders, D.A., Hudson, A.D., Tewkesbury, G.E. and Mason, S. (2000), "Automating the design of high-recirculation airlift reactors using a blackboard framework", Expert Systems with Applications, Vol. 18 No. 3, pp. 231-45.

Sanders, D.A., Tan, Y.C., Rogers, I. and Tewkesbury, G.E. (2009b), "An expert system for automatic design-forassembly", Assembly Automation, Vol. 29 No. 4, pp. 378-88.

Sanders, D.A., Urwin-Wright, S.D., Tewkesbury, G.E. and Gremont, B. (2005), "Pointer device for thin-film transistor and cathode ray tube computer screens", Electronics Letters, Vol. 41 No. 16, pp. 894-6.
Sanders, D.A., Hudson, A.D., Cawte, H., Fenske, O., Poland, G.A. and Tewkesbury, G.E. (1994), "Computer modelling of single sludge systems for the computer-aideddesign and control of activated sludge processes", Microprocessing and Microprogramming, Vol. 40 Nos 10-12.

Stahl, F., Bramer, M. and Adda, M. (2009), "PMCRI: a parallel modular classification rule induction framework", Proceedings of the 6th International Conference on Machine Learning and Data Mining in Pattern Recognition, Berlin, Vol. 5632, pp. 148-62.

Stott, I.J. and Sanders, D.A. (2000a), "New powered wheelchair systems for the rehabilitation of some severely disabled users", International fournal of Rehabilitation Research, Vol. 23 No. 3, pp. 149-53.

Stott, I.J. and Sanders, D.A. (2000b), "The use of virtual reality to train powered wheelchair users and test new wheelchair systems", International fournal of Rehabilitation Research, Vol. 23 No. 4, pp. 321-6.

Stott, I.J., Sanders, D.A. and Goodwin, M.J. (1997), "A software algorithm for the intelligent mixing of inputs to a tele-operated vehicle", Fournal of Systems Architecture, Vol. 43 Nos 1-5, pp. 67-72.

Tewkesbury, G.E. and Sanders, D.A. (1994), "The automatic programming of production machinery for de-flashing plastic parts", Advances in Manufacturing Technology, Vol. VIII, pp. 279-83.

Tewkesbury, G.E. and Sanders, D.A. (1999a), "A new robot command library which includes simulation", Industrial Robot, Vol. 26 No. 1, pp. 39-48.

Tewkesbury, G.E. and Sanders, D.A. (1999b), "A new simulation based robot command library applied to three robots", fournal of Robotic Systems, Vol. 16 No. 8, pp. 461-9.

Tewkesbury, G.E. and Sanders, D.A. (2001), "The use of distributed intelligence within advanced production machinery for design applications", Total Vehicle Technology: Challenging Current Thinking, Department of Mechanical and Manufacturing Engineering, University of Portsmouth, Portsmouth, pp. 255-62.

Urwin-Wright, S.D., Sanders, D.A. and Chen, S. (2002), "Terrain prediction for an eight-legged robot", fournal of Tobotic Systems, Vol. 19 No. 2, pp. 91-8.

Urwin-Wright, S.D., Sanders, D.A. and Chen, S. (2003), "Predicting terrain contours using a feed-forward neural network", Engineering Applications of Artificial Intelligence, Vol. 16 Nos 5/6, pp. 465-72.

\section{Corresponding author}

David A. Sanders can be contacted at: david.sanders@ port.ac.uk 